\title{
Tendencias en los sistemas educativos del siglo XXI
}

\author{
Trends in Education Systems in the 21st Century
}

\section{Tendências dos sistemas de educação do século 21}

\author{
Jayson AndRey Bernate* \\ * Doctorando en Ciencias de la Educación. Docente Investigador de la Corporación \\ Universitaria Minuto de Dios, UNIMINUTO. Bogotá, Cundinamarca, Colombia https:// \\ orcid.org/0000-0001-5119-8916
}

\section{OPEN ACCESS \\ DOI: http://dx.doi.org/10.18634/sophiaj. 17v.1i.1015}

Información del artículo

Recibido: octubre 06 de 2020

Revisado: enero 12 de 2021

Aceptado: enero 28 de 2021

Publicado: abril 15 de 2021

Palabras clave: educación, sistema educativo tendencia educacional, tecnología, .

Keywords: educational trend, educational system, technology, education.

Palavras-chave: tendência educacional, Sistema educacional, tecnologia, educao

Cómo citar: /how cite:

Bernate, J.A. (2021) Tendencias en los sistemas educativos del siglo XXI. Sophia 17 (1), e1015

Sophia-Educación, volumen 17 número 1. enero/junio 2021. Versión español

\section{RESUMEN}

El presente artículo de reflexión teórica surge desde el espacio académico de tendencias y desafíos de los sistemas educativos del Doctorado en Ciencias de la Educación de la Universidad Metropolitana de Educación Ciencia y Tecnología (Umecit), el cual tiene por propósito considerar los aspectos conceptuales de las tendencias educativas del siglo XXI en diferentes contextos. La metodología desarrollada fue la cualitativa-reflexiva, los principales hallazgos están enfocados hacia la explicación de las principales metodologías y didácticas utilizadas en Latinoamérica, ubicando a los actores directamente implicados en el acto educativo como potenciadores de los sistemas sociales y culturales de una comunidad emergente de conocimiento y necesidades propias, generando transformaciones a nivel político y gubernamental en las naciones. A modo de conclusión de esta reflexión académica es que los actores educativos de las pedagogías emergentes de aprendizaje tecnológico, deben estar constantemente sumergidos e integrados en la formación profesional y académica, ya que así probablemente podrán responder a los retos y necesidades de la sociedad y comunidad actual en las diferentes instituciones educativas. 
Copyright 2021 Universidad La Gran Colombia

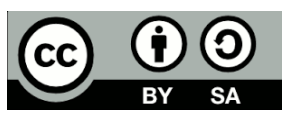

Conflicto de interés:

Los autores declaran no tener ningún conflicto de interés.

Correspondencia de autor:

jbernate1@uniminuto.edu.co

\section{ABSTRACT}

This article of theoretical reflection arises from the academic space of trends and challenges of educational systems of the Doctorate in Educational Sciences of the Metropolitan University of Education, Science and Technology (UMECIT), which aims to consider the conceptual aspects of the educational trends of the XXI century in different contexts. The methodology developed was qualitative-reflexive. The main findings are focused on the explanation of the main methodologies and didactics used in Latin America, locating the actors directly involved in the educational act as promoters of the social and cultural systems of an emerging community of knowledge and own needs, generating transformations at political and governmental level in the nations. As a conclusion of this academic reflection, the educational actors of the emerging pedagogies of technological learning must be constantly immersed and integrated in the professional and academic training, since this way they will probably be able to respond to the challenges and needs of the current society and community in the different educational institutions.

RESUMO

Este artigo de reflexão teórica surge do espaço acadêmico de tendências e desafios dos sistemas educacionais do Doutorado em Ciências da Educação da Universidad Metropolitana de Educación ciencia y Tecnología (UMECIT), que visa considerar os aspectos conceituais das tendências educacionais do século XXI em diferentes contextos. A metodologia desenvolvida foi qualitativo-reflexiva, os principais achados estão voltados para a explicação das principais metodologias e didáticas utilizadas na América Latina, localizando os atores diretamente envolvidos no ato educativo como potencializadores dos sistemas sociais e culturais de uma comunidade emergente de conhecimento. e próprias necessidades, gerando transformações no nível político e governamental nas nações. A conclusão desta reflexão acadêmica é que os atores educacionais das pedagogias de aprendizagem tecnológica emergentes devem estar constantemente imersos e integrados na formação profissional e acadêmica, pois assim provavelmente serão capazes de responder aos desafios e necessidades da sociedade e da comunidade atual em diferentes instituições de ensino. 


\section{Introducción}

Es indudable que la educación en todos sus niveles requiere una transformación social, sin embargo, cabe resaltar diez tendencias del siglo XXI, las cuales como educadores se deben tener presente para dicha transformación; alumnos empoderados, personas felices y sanas, aprendizaje permanente, espíritu emprendedor, programar, inclusión de tecnología, gamificación, responsabilidad social, poder colectivo y Ágora.

Por otro lado, es importante mencionar que lo anterior debe ir articulado con el presupuesto de una nación, estas mejoras a nivel económico generan que los niveles de aprendizaje en básica primaria, secundaria, universitaria y adultos extra edad se optimicen en todos los ámbitos sociales y culturales, sin embargo, cabe destacar que una adecuada infraestructura educacional genera mejoras pedagógicas y curriculares en los procesos de calidad del aprendizaje, no dejando de lado la constante capacitación docente, vinculando dichos aportes económicos y presupuestales a la transformación de leyes y decretos que permitan que esto no se quede en el papel, sino que verdaderamente sea llevado a las aulas del diario vivir en las diferentes instituciones educativas. Tal como señalan Melo, Ramos \& Hernández (2017):

Para lograr un mejoramiento de los resultados académicos, las medidas de política del Estado y las estrategias de las instituciones deben tomar en cuenta, además de los criterios en la contratación de docentes, la definición de incentivos para la investigación y los aspectos administrativos y financieros, los mecanismos que permitan ayudar a contrarrestar el impacto negativo derivado de las condiciones socioeconómicas de los estudiantes y de otros factores ambientales. (p. 103)

A partir de las transformaciones educativas en las diferentes naciones, surgen las necesidades de ser líderes en el siglo XXI, pero ello implica enfrentarse a retos sociales, culturales, generacionales, étnicos; pero sobre todo tecnológicos, ya que este último cambia a pasos agigantados día a día y la educación en todos sus niveles se debe adecuar a estos cambios, puesto que requiere influir a los actores dinámicos de la sociedad, hoy día no se trata de transferir conocimientos, sino en ser un influenciador de pensamiento y aprendizaje dinámico dentro del marco pedagógico y didáctico, las instituciones educativas y docentes deben ir más allá del simple acto enseñanza-aprendizaje, puesto que estas formas son cambiantes y fluctuantes, ya que los jóvenes de hoy no leen, en vez de esto googlean y youtubean, por tal motivo, los componentes curriculares y metodológicos de la enseñanza deben sufrir adecuaciones y transformaciones severas para que se adecuen a las necesidades de la realidad social que viven los actores directamente implicados en el acto educativo. Como recomiendan Hernández, Gómez \& Balderas (2014):

El proceso de inclusión de TIC en las actividades curriculares debe acompañarse de la creatividad para diseñar las actividades por realizar, con el fin de lograr la atención, motivación y participación activa del estudiante en el desarrollo de la clase, teniendo en cuenta que es el docente el gestor de los nuevos ambientes de aprendizaje, el encargado de crearlos e innovarlos, incorporando los medios tecnológicos. (p. 18)

\section{Tendencias tecnológicas en el siglo XXI}

Un ejemplo claro de las tendencias tecnológicas en el aula es el modelo de gamificación, el cual utiliza el juego como herramienta para el desarrollo conceptual de los estudiantes en el aula y cualquier nivel educativo puede aplicar para esto. Explicado desde el modelo R.A.M.P. (Modelo de las motivaciones intrínsecas y extrínsecas) creado por Andrezj Marczewski, el cual consiste en desarrollar en los estudiantes cuatro elementos inherentes en el proceso de aprendizaje las actividades educativas, pero utilizando el juego como elemento primario en pro de conseguir los saberes que deben potenciar los mismos, a partir del interés y motivación que tenga cada uno, esto conlleva a que sin darse cuenta el estudiante desarrolle los siguientes elementos: relación, la cual consiste en generar sentido de pertenencia en lo que desarrolla, estatus social, conexiones y compartir. Como segundo elemento, el propósito, el cual forma y potencia el altruismo y sentido común en las acciones de juego para que pueda ayudarse a sí mismo y a los demás. El tercer elemento, es la muestra, la cual consiste en aprender y mejorar su desempeño en algo específico y así lograr un alto desempeño personal. Como último elemento, la autonomía, la cual genera en el estudiante libertad de creatividad en sus tareas, pero cumpliendo roles de responsabilidad y control kinestésico.

Como se observa el A.B.J (Aprendizaje basado en el juego) potencia muchos elementos en la población estudiantil y como tendencia educativa en el siglo XXI enmarca una opción para los educadores modernos, puesto que sirve como estrategia pedagógica para responder a los retos sociales y culturales de la sociedad y es una invitación a los estudiantes a que escojan el camino más difícil y no el más fácil, puesto que si escogen 
el más difícil los resultados de aprendizajes serán mucho más significativos. Tal como proponen Zepeda, Abascal \& Omelas (2016):

La gamificacion ayuda a realizar reflexión sobre la didáctica que se usa en una clase y experimentar con nuevas formas lúdicas que permitan mejorar y hacer adecuaciones en la conducción de grupo, ideas de presentación de teoría, actividad práctica, considerar nuevas formas de evaluar y consecuentemente mejorar el rendimiento escolar. (p. 323)

Otra tendencia muy utilizada por los pedagogos modernos es el modelo de aula invertida flip classroom el cual articula los procesos en las tendencias del siglo XXI, este se origina en los años noventa y surge a partir de necesidades de la sociedad de la época y contiene cuatro pilares; el primero, es el de ambiente flexible, el cual consiste en que la institución educativa cuente con áreas amplias específicas de aprendizaje y pueda desarrollarse plenamente en cada una de ellas; la segunda, es la cultura de aprendizaje y esta consiste en que el estudiante no aprenda por una nota o por un porcentaje o peso académico, sino que por el contrario encuentre interés propio en cada actividad; el tercero, es el contenido dirigido y este consiste en que el rol del maestro sea activo dentro del aula y genere liderazgo en las temáticas de interés de los estudiantes y no coarte las temáticas ni tampoco las segmente; por último, el facilitador profesional, el cual busca siempre la evolución del aprendizaje y que este se encuentre en constante cambio en pro de las mejoras a diario. Es un modelo integral, dinámico y eficaz el cual enmarca un aprendizaje integral dentro y fuera del aula, por tal motivo, Torrecilla (2018) recomienda: “En comparación con el modelo más tradicional, el docente trabaja de forma más cercana con los estudiantes y puede tener un conocimiento más amplio de cuáles son sus necesidades y habilidades, personalizando el proceso enseñanza-aprendizaje" (p. 18).

En consecuencia con ello, el docente canadiense Ken Baver del Tecnológico de Monterrey sugiere cuatro claves para que el modelo se cumpla plenamente, las cuales consisten en el aprendizaje activo; no es necesario que el maestro tenga el control del aula, el siguiente es el de conocer a sus estudiantes, es decir, que se interactúe más con ellos, conocer sus gustos, sus miedos, sus sueños, sus trabajos, etc. La tercera clave es el conectivismo global y en este hace hincapié en que los estudiantes compartan sus tareas, sus ideas principales y sus consultas bibliográficas por medio de blogs y redes sociales académicas navegantes dinámicas; por último, el de confiar en los estudiantes, recomienda quitar impedimentos y potenciar la autoevaluación como proceso inherente del aprendizaje.

\section{Aprendizaje basado en retos}

El acto de educar es algo complejo y sistémico, demanda un compromiso no solo de los actores educativos, sino de todo un sistema político y social, por ello, las tendencias modernas son tan importantes para sostener y articular esta complejidad, cabe citar el aprendizaje basado en retos como una tendencia representativa en el mundo actual y postmodernista, ya que es un sistema que implica estímulos y emociones que conllevan al estudiante a un desafío para llevarse a cabo, lo involucra activamente en una situación problemática real y relevante vinculando el entorno como reto desarrollando una implementación de una solución. Así es como Olivares et al. (2018) definen el ABR como la: "Oportunidad de aprendizaje en la que los estudiantes colaboran, bajo la guía del profesor, para aprender sobre problemas relevantes mediante la propuesta de soluciones reales" (p. 232).

Cabe señalar que este aprendizaje es basado en el funcionamiento esquemático del mundo real en el hacer y actuar centralizado en el estudiante, puesto que articula experiencias de vida y desarrolla los intereses particulares y significativos para darle un significado práctico a la educación, mientras se potencian competencias como el trabajo colaborativo y multidisciplinario, la toma de decisiones, la ética y el liderazgo. Convirtiéndolo en una tendencia innovadora del siglo XXI, como opción para los sistemas políticos y educativos de la actualidad. Tal como sustenta Ramírez (2020):

La innovación educativa aparece como un tema pendiente en las agendas de las instituciones educativas a nivel internacional. Las políticas educativas vigentes ponen de manifiesto la importancia de la innovación educativa como un elemento clave para la mejora de la calidad educativa y el desarrollo social. (p. 2)

Algunos de los beneficios para los estudiantes en dicho aprendizaje son que se involucran tanto en la definición del problema a ser abordado como en la solución que desarrollarán para resolverlo. Logrando una comprensión más profunda de los temas, para así aprender a diagnosticar pertinentemente los problemas antes de proponer soluciones, así como también desarrollan creatividad, generando sensibilización ante una situación dada, 
innovando procesos investigativos innovadores y científicos para su posterior materialización, respondiendo los retos de la sociedad. Como propone Waldman \& Gurovich (2005):

Dar respuesta a los desafíos que plantea una sociedad cada vez más heterogénea, que exige mayor eficiencia en la formación de sus ciudadanos, y que, al mismo tiempo, reclama un sistema universitario de calidad, basado en el derecho a la igualdad de oportunidades. (p. 22)

Indudablemente desde las instituciones educativas se deben generar esos apalancamientos bien cimentados en pro de mejorar la calidad de los procesos de enseñanza-aprendizaje, convirtiendo y transformando la sociedad, es decir, que esos estudiantes que se están formando en los diferentes programas de pregrado y posgrado respondan a los retos que demanda la sociedad de manera oportuna y eficaz. Tal como lo expone Enríquez (2006): “Que los estudiantes salgan de la universidad portando no solo sus diplomas de graduación sino también, conocimiento relevante para vivir en sociedad, junto con la destreza para aplicarlo y adaptarlo a un mundo en constante cambio" (p. 12).

\section{Gestión educativa}

Lo que se necesita en la actualidad en las instituciones educativas formadoras de pregrado y posgrado es que se alimente la actualización permanente de los programas y mallas curriculares, que respondan a las verdaderas necesidades de la sociedad actual y así evitar las constantes fracturas entre el puente directo de educación y vida sociocultural. En el siglo XXI, la única manera de fortalecer la identidad y autodeterminación de un país es por medio de la educación, este es el único camino.

Por otra parte, es preciso mencionar los aspectos negativos que influyen en el desarrollo e impacto de las tendencias educativas en Latinoamérica, uno de los más influyentes en dicho proceso es la privatización, este fenómeno ha sido potenciado y enmarcado desde el modelo neoliberal de los sistemas políticos, lo cual ha generado que la brecha social de desigualdad sea más amplia en los jóvenes de bajos recursos, los cuales deben solicitar créditos económicos a largo plazo con intereses muy altos para poder acceder a la educación superior, estas dificultades las cita Linares (2016) como: "El entorpecimiento en las normatividades, requisitos de ingreso, programas, enfoques, tendencias y los modelos que han desarrollado estos países para su adaptación hacia los modelos neoliberales y de globalización." (p. 43).

La característica primordial de todo estudiante al poder acceder a un servicio de educación superior es ciertamente satisfactoria, sin embargo, cuando este culmina el proceso se convierte en un egresado del servicio prestado y en cierta medida entra a ser un evaluador del impacto del programa y su incidencia en la calidad del mismo, juzgando la inversión y el tiempo del mismo, dándole respuesta alguna al horizonte competitivo laboral como explica Villanueva (2020):

El grado de la percepción del usuario que tiene sobre el servicio es lo que determina la calidad de este y si la adquisición de conocimiento en una institución en específico otorga o no un reconocimiento social por contar con un factor diferenciador que indica que este servicio es de calidad. (s.f)

Por tal motivo, cuando se apunta hacia sistemas educativos de calidad es importante y pertinente generar estrategias de gestión en la formación y actualización docente, en cuanto se potencien sus habilidades pedagógicas y metodológicas en pro de masificar su acto educativo en las aulas y sobre todo fuera de ellas, para poder brindar una educación de calidad e integral en todos sus procesos, para poder acercarse al objetivo y meta ideal en formar profesionales que puedan responder a las necesidades de la sociedad actual. Tal y como socializa Rico (2016):

La gestión de procesos de formación docente es necesario en cualquier institución con miras a tener una educación con calidad, y no solo eso, la exigencia del mercado hace que las universidades además de tener excelente infraestructura, requieren que se le otorgue valor a la labor de los docentes, no solo de tipo económico, también, valor a la labor del profesor es garantizar las oportunidades de su profesionalización, formación y desempeño dentro de la universidad, de esta forma, se está ahondado en las necesidades del grupo docente y abarcando las necesidades de la institución y su meta de alcanzar una educación de calidad. (p. 58)

Es imperante comenzar a trabajar directamente en la gestión de los procesos educativos, ya que es allí donde se planifican los alcances e impacto de las necesidades educativas, por tal motivo, el liderazgo escolar cumple un papel preponderante en estas acciones de mejora continua en dichos procesos: "Destacando la optimización y ampliación de las infraestructuras, desarrollo de nuevos Programas Educativos, mayor 
implicación de las familias, mejoras organizativas en la planificación, trabajo en equipo, TIC, formación del profesorado, etc." (Rodríguez, Ordóñez y López, 2020, p. 289).

Convirtiéndose lo anterior en reformas educativas que atañen los sistemas sociales, generando a su vez ciertas tensiones a nivel político y gubernamental, como es el caso de muchos países latinoamericanos, especialmente en Colombia, donde lamentablemente los recursos económicos se desvían y los escándalos del recurso público están a la orden del día, originando en la sociedad mucho malestar y desazón hacia estos sistemas políticos (Castaño \& García, 2012).

Reforzando lo anterior Waldman (2000) recomienda que: "El principal desafío de la educación superior consiste en transformarse institucional y académicamente a fin de satisfacer, de manera oportuna y eficiente, las demandas de una sociedad globalizada que exige una formación permanente." (p. 241). El desafío de la educación no es solo abordar problemáticas sociales, educativas y culturales, va mucho más allá y se quiera o no, se articulan con lo político, por cuestiones de presupuesto y facilidades normativas que implican reformas constitucionales en cada país. Por ello es pertinente que como nación se estimulen a los diferentes actores del contexto educativo, a que transformen sus comunidades en pro del avance local, regional y nacional de las instituciones, tal como lo recomiendan Álvarez et al. (2015) “Un líder educativo debe, como primera instancia, tener los conocimientos fundamentales en cuestiones administrativas que sustenten su gestión adecuada, mirando a la escuela como una organización que requiere una proyección institucional pertinente." (p. 66).

La importancia de tener líderes preparados y formados en educación es un factor determinante a la hora de exigir resultados óptimos y acordes con las necesidades de todas las instituciones, ya que son quienes marcarán la línea de partida y la ruta a seguir para articular todo el trabajo pedagógico y metodológico de la instituciones y de esto va a depender el éxito o el fracaso del mismo. Como proponen Casanoves, Küster y Vila (2019):

Por tanto, se considera que los gerentes deben mejorar sus servicios e instalaciones $\mathrm{y}$, a posteriori, incentivar a capital interno con programas de formación y mejora salarial, para que éstos desarrollen con más pasión su trabajo, sean más innovadores y el resultado pueda percibirse por parte de los estudiantes. (p. 123)

Ademas de las demandas que solicita hoy el servicio educativo, no hay que dejar de lado la época digital que se está viviendo, ya que es una influencia determinante en pro de conseguir los resultados a que deben llegar las tendencias educacionales de hoy; así como lo sustentan Ricoy y Martínez (2020): "La sociedad del conocimiento exige nuevos desafíos que deben ser afrontados de modo resolutivo y teniendo en cuenta el dominio que ejerce la tecnología digital, particularmente en los/ as adolescentes." (p.479). Por tal motivo, los órganos legales y estatales que rigen estos sistemas educativos deben ser conscientes de la premura que exige tal fenómeno.

No se puede dejar de lado la inclusión como factor urgente de integración y articulación a todos los procesos gestacionales y de planificación educativa, ya que muchas instituciones en Latinoamérica hasta hace poco están repensando en cómo hacer para poder responder a esta necesidad social, es allí donde otra tendencia como la equidad entra en el actuar asertivo de los sistemas educativos, tanto en la parte pedagógica como en la parte administrativa y legislativa, como deducen Sánchez y Manzanares (2013):

La equidad como inclusión educativa, con énfasis en la redistribución de recursos educativos, generación de situaciones de aprendizaje ajustadas a los ritmos y expectativas de aprendizaje del alumnado desde un enfoque inclusivo. A los inputs y outputs se les empieza a considerar elementos de equidad implicados en el proceso educativo. De este modo, se entiende que la educación debe ser de calidad para todos en un contexto de equidad, donde la cohesión social ha de regir la función y misión de los sistemas educativos. (p. 24-25)

Al mismo tiempo la investigación educativa debe ser un papel dinámico dentro del acto educativo, pues es el docente quien debe marcar el camino al estudiante en todos sus niveles de una manera eficiente, lo cual demandará un compromiso reflexivo y responsable para la consecución de la transformación histórica y social, para lograr una optimización de la calidad de vida (Hernández, 2009).

Todo conlleva a que el proceso de enseñanza-aprendizaje sea más integral y representativo en cuanto a lo social, para que los actores pedagógicos lo vivencien y comprendan el verdadero sentido de educar (Bernate, García, Fonseca \& Ramírez, 2020). 


\section{Conclusiones}

Las tendencias educativas del siglo XXI deben estar articuladas con los sistemas políticos y gubernamentales de los países, si estos van en contravía va a ser muy difícil que sean efectivas y puedan responder a las necesidades sociales de las comunidades a educar.

Los planes curriculares en los componentes pedagógicos deben romper los paradigmas establecidos durante la historia de su gestación, para que puedan sufrir transformaciones pertinentes a las necesidades de los actores sociales de la comunidad educativa y lograr un impacto positivo en las necesidades propias.

Las tecnologías de la información deben estar al servicio del cuerpo docente, estudiantado y personal administrativo, para que se pueda llegar a todos y cada uno de los implicados en los procesos de enseñanzaaprendizaje, sumergiéndose constantemente en la actualización de estrategias modernas y didácticas, generando la motivación e interés de aprender, transformando los significados del aula, ya que sin emociones no habrá aprendizaje.

El presupuesto económico para la educación en los países latinoamericanos debe ser una inversión mayoritaria, respecto a los otros intereses gubernamentales, puesto que si no se atiende con compromiso y dedicación a la sociedad, más tarde estos podrán convertirse en problemáticas sociales irreversibles.

Despertar el emprendimiento con sentido de responsabilidad social en la comunidad educativa, puede desembarcar en un país el desarrollo cultural, social, económico y político, que a su vez adhiere consecuencias positivas hacia la nación llevándola a la constante evolución y transformación de sus sistemas legislativos y gubernamentales optimizando el desarrollo crítico social del mismo.

\section{Referencias bibliográficas}

Álvarez Botello, J., Torres Velázquez, A. M., \& Chaparro Salinas, E. M. (2015). Diagnóstico del liderazgo educativo en las Instituciones de Educación Superior del Valle de Toluca. Revista De Investigación Educativa, 34(1), 51-68. https://doi.org/10.6018/rie.34.1.206881

Bernate, J. A., García-Celis, M. F., Fonseca-Franco, I. P., \& Ramírez-Ramírez- N. E. (2020). Prácticas de enseñanzas y evaluación en una facultad de educación colombiana. Rev.investig.desarro.innov., 10 (2). Doi: 10.19053/20278306.v10.n2.2020.10721

Castaño-Duque, G. A., \& García-Serna, L. (2012). Una revisión teórica de la calidad de la educación superior en el contexto colombiano. Educación y Educadores, 15(2), 219-243., from http://www.scielo.org.co/scielo. php?script=sci arttext\&pid=S0123-12942012000200005\&lng=en\&tIng=es

Casanoves Boix, J., Küster Boluda, I., \& Vila López, N. (2018). ¿Por qué las instituciones de educación superior deben apostar por la marca? Revista De Investigación Educativa, 37(1), 111-127. https://doi.org/10.6018/ rie.37.1.291191

Enríquez Clavero, J (2006). Educación superior: tendencias y desafíos. Educación Médica, 9 (1), 06-10. Recuperado de http://scielo.isciii.es/scielo.php?script=sci arttext\&pid=S1575-18132006000100003\&l$\underline{n g}=e s \& t \operatorname{lng}=e s$.

Hernández Arteaga, I (2009). El docente investigador en la formación de profesionales. Revista Virtual Universidad Católica del Norte, (27) ,1-21.. Recuperado de: https://www.redalyc.org/articulo. oa?id=1942/194215432011

Hernández Doria, C, \& Gómez Zermeño, M, \& Balderas Arredondo, M (2014). Inclusión de las tecnologías para facilitar los procesos de enseñanza - aprendizaje en ciencias naturales. Revista Electrónica "Actualidades Investigativas en Educación", 14(3) ,1-19.. Recueprado de https://www.redalyc.org/articulo. oa?id $=447 / 44732048010$

Melo-Becerra, L. A., Ramos-Forero, J. E., \& Hernández-Santamaría, P. O. (2017). La educación superior en Colombia: situación actual y análisis de eficiencia. Desarrollo y Sociedad, 2017(78), 59-111. doi:10.13043/ DYS.78.2 
Olivares Olivares, S. L., López Cabrera, M. V., y Valdez-García, J. E. (2018). Aprendizaje basado en retos: una experiencia de innovación para enfrentar problemas de salud pública. Educación Médica. 19, 230-237 doi:10.1016/j.edumed.2017.10.001

Ramírez Ramírez, L (2020). Tendencias de la innovación educativa en los contextos sociales. Análisis del mapeo de literatura. Revista Educación, 44(1),1-27..Recuperado de: https://www.redalyc.org/articulo. oa? id $=440 / 44060092001$

Rico, A.D. (2016) La gestión educativa: Hacia la optimización de la formación docente en la educación superior en Colombia. Sophia 12(1): 55-70.

Ricoy, M-C., y Martínez-Carrera, S. (2020). El uso informal del Smartphone en adolescentes de centros de protección: un reto para promover la intervención socioeducativa. Educación XX1, 23(1), 459-482, doi: 10.5944/ educXX1.23879

Rodríguez-Gallego, M., Ordóñez-Sierra, R. y López-Martínez, A. (2020). La dirección escolar: Liderazgo pedagógico y mejora escolar. Revista de Investigación Educativa, 38(1), 275-292. DOI: http://dx.doi.org/10.6018/ rie.364581

Sánchez Santamaría, J. y Manzanares, A. (2013). Tendencias internacionales sobre equidad educativa desde la perspectiva del cambio educativo. Revista Electrónica de Investigación Educativa, 16(1), 12-28. Recuperado de http://redie.uabc.mx/vol16no1/contenido-sanchez-manzanares.html

Torrecilla Manresa, S. (2018). Flipped Classroom: Un modelo pedagógico eficaz en el aprendizaje de Science. Revista Iberoamericana De Educación, 76(1), 9-22. https://doi.org/10.35362/rie7612969

Villanueva-Vázquez, A. (2020). Modelo exploratorio de calidad en la educación superior. Dimensión Empresarial, 18(1). DOI: 10.15665/dem.v18i(1).2239

Waldman Mitnick, G (2000). Los rumbos de la educación superior: tendencias y desafíos. Revista Mexicana de Ciencias Políticas y Sociales, XLIV (180), 227-243. Disponible en: https://www.redalyc.org/articulo. oa? id $=421 / 42118016$

Waldman M., G, \& Gurovich, L. (2005). Tendencias, desafíos y oportunidades de la educación superior al inicio del siglo XXI. Universidades, (29) ,13-22. Recuperado de https://www.redalyc.org/articulo. oa? id $=373 / 37302903$

Zepeda - Hernández, S, \& Abascal - Mena, R, \& López - Ornelas, E (2016). Integración de gamificación y aprendizaje activo en el aula. Ra Ximhai, 12(6) 315-325.. Recuperado de https://www.redalyc.org/articulo. oa?id=461/46148194022 